\title{
A statistical model for maximum avalanche run-out distances in southwest Montana
}

\author{
L. R. MCKITTRICK AND R. L. BROWN \\ Department of Civil and Agricultural Engineering, Montana State University, Bozeman, MT 59715, U.S.A.
}

\begin{abstract}
An extreme value (Gumbel) distribution was evaluated as a model of maximum run-out distances of avalanches in southwest Montana defined in terms of dimensionless ratios. The run-out ratios are defined relative to an arbitrarily defined reference point. The development and analysis of the statistical model were based on data taken from surveys of 24 avalanche paths. The technique bears promise as a user-friendly tool; however, due to the small vertical drop of avalanches in southwest Montana, the model appears to be quite sensitive to measurement errors in the field. This sensitivity also seems to depend on the definition of the reference point. Comparison with models developed for other mountain ranges indicates that models developed for one range cannot be used to estimate accurately maximum avalanche run-out distances in other mountain ranges until influencing factors are better understood.
\end{abstract}

\section{INTRODUCTION}

To determine where structures can be built safely, it is desirable to estimate maximum avalanche run-out distances or "100-year" avalanche zones. Voellmy's fluid model and the unified center-of-mass model have often been considered the standards for determining run-out distances for avalanches, but the necessary parameters are often difficult to obtain without an intuitive feel.

Recently more sophisticated methods of modeling avalanche dynamics, reviewed by Lang and Dent (1982) have been developed as a means of estimating avalanche run-out distances. These methods initially modeled a flowing avalanche as laminar flow of a Newtonian fluid (Lang and others, 1979; Lang and Brown, 1980). Numerous field evaluations (Martinelli and others, 1980) verified that this computational model could be used to predict avalanche run-out distances accurately, but reasonable values for the material viscosity and friction coefficient had to be determined. This model still required user experience to estimate the required parameters. In addition, the assumption of laminar flow left questions regarding the ability of the model to duplicate accurately some details of the flow.

Dent and Lang (1982) later improved on the earlier model by representing the fluid as a biviscous material. This model gave very good estimations of run-out distance, avalanche speed and debris-pile geometry when compared with actual data. Dent and Lang's computational model, again, required the user to estimate some material parameters.

More recent studies on avalanche dynamics include those of Hutter (1989) and Lang (1991). These models employ very sophisticated theories of turbulent granular material flow. Their results look very promising, and continued development of these formulations offers promise of powerful formulations for predicting many details of avalanche dynamics, including run-out distances.

The purpose of this research is to develop a more userfriendly tool for estimating maximum avalanche run-out distances in southwest Montana. The method of McClung and others (1989) offers an ideal means of achieving this goal. This method is essentially a statistical evaluation of historical avalanche activity in any given mountain range. Maximum run-out distances are estimated avalanche extremes that have occurred in the last 100-year period. McClung has applied this approach to mountain ranges in Norway, the Sierras, the Colorado Rockies and the Canadian Rockies, and has noted substantial discrepancies in the results for these ranges. These discrepancies may be due to differences in terrain features and to weather conditions. The great majority of slide paths evaluated by McClung were large, over $350 \mathrm{~m}$ vertical drop. In this study we tested the applicability of McClung's approach to the mountains in southwest Montana where a large percentage of the slide paths is considerably smaller.

Maximum avalanche run-out distances were measured on 24 avalanche paths in the Madison and Gallatin ranges of southwest Montana. Slope and distance measurements were taken with an inclinometer and tape measure. Maximum run-out distances were determined using records of past avalanches in terms of tree destruction or other types of vegetation damage. In several cases the relative frequency of different avalanche run-out distances was clearly displayed as a series of steps in tree ages. In these cases only younger trees grew in the 
run-out zones of the more frequent avalanches, while slightly older trees grew in the extended run-out zones of the larger but less frequent avalanches.

Since avalanche run-out distances are known to have a probabilistic nature, the data collected was used to construct a statistical model of maximum run-out distances. The model is based on the ratio of two distance parameters which can be easily determined from field measurements.

The statistical approach taken by McClung and others (1989) is based on the definition of a dimensionless run-out ratio, which is then used as the random variable of a probability distribution. Before defining the dimensionless run-out ratio, a reference point must be selected along the avalanche path, as shown in Figure 1. McClung and others define their reference point, $\beta$, as the first point along the avalanche path that diminishes to a specified local slope angle, $\theta_{\beta}$. This definition can be applied fairly consistently if the paths are sufficiently worn so as to be continuously concave; but if the path is interrupted by ledges or sloping benches, then the reference point may change position as a function of the averaging or smoothing technique used to define local slope angles. Some of these ledges are so small that they have an insignificant influence on the avalanche run-out distance and they can be modeled by smoothing out the discontinuity and choosing the next lower position which meets the definition of the reference point. If the slope is interrupted by a larger bench with a shallow slope and the reference point is positioned on this bench, the probabilistic model, developed using continuously concave slopes, appears to lead to an underestimation of the expected maximum run-out distance (McClung and Lied, 1987).

The run-out ratio is defined as the horizontal distance, $\Delta x$, between the $\beta$ point and the extreme run-out position divided by the horizontal distance, $x_{\beta}$, between the $\beta$ point and the starting position of the avalanche. In symbolic terms, we have

$$
\text { run-out ratio }=\frac{\Delta x}{x_{\beta}} .
$$

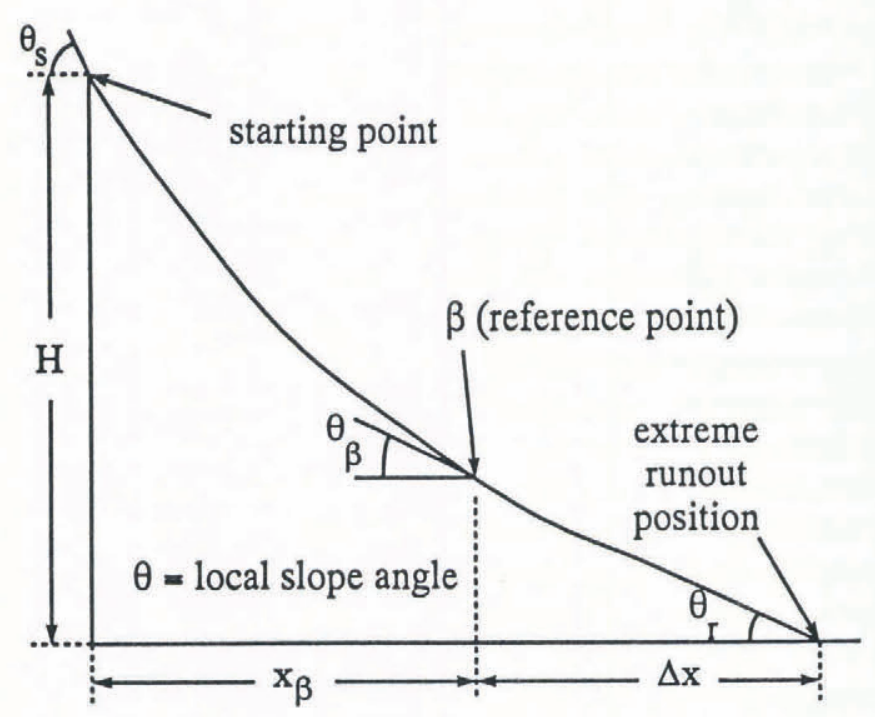

Fig. 1. Parameters defined in terms of path geometry.
When we first began this study, we intended simply to extend or confirm the accuracy of the statistical model used by McClung and others (1989); however, we chose smaller avalanche paths and hence had to alter their model slightly. McClung and others defined their $\beta$ point where the local slope angle, $\theta$, first diminished to $10^{\circ}$, but out of the 24 avalanche paths we surveyed, only 5 ran far enough to reach a local slope angle of $10^{\circ}$. In some cases, $\theta$ did not diminish to $10^{\circ}$ for 200 or $300 \mathrm{~m}$ beyond the end of the extreme run-out position; therefore, we felt that a $10^{\circ}$ reference point had no physical significance relative to our paths and decided to search for a new definition for the $\beta$ point.

To define the path profile, we surveyed the avalanche paths by taking slope and distance measurements every $50 \mathrm{~m}$ in regions where the slope was relatively constant. We shortened our survey distance where the slope was highly variable. As mentioned previously, since local regions of the slope can display significant variability, the location of the $\beta$ point can be quite dependent on the averaging technique that is applied. To simplify the averaging process and keep it as consistent as possible, we decided to model each avalanche slope using the leastsquares fit second degree polynomial. This approach also allowed us easily to vary the definition of $\theta_{\beta}$ and solve for new $\beta$ points on each avalanche path.

McClung and Lied point out that an extreme value (Gumbel) distribution,

$$
p=\exp \left(-\exp \left(-\frac{\frac{\Delta x}{x_{\beta}}-u}{b}\right)\right),
$$

provides a good model of maximum avalanche run-out distances where the dimensionless run-out ratio is the random variable and $u$ and $b$ are the location and scale parameters, respectively. In this case, $p$ is the nonexceedance probability divided by 100 (i.e. $0 \leq p \leq 1$ ).

To obtain estimates for the parameters using our data, we rewrite the previous equation as

$$
\begin{aligned}
\left(\frac{\Delta x}{x_{\beta}}\right)_{i} & =u-b \ln \left(-\ln \left(p_{i}\right)\right) \\
& =u+b Y_{p},
\end{aligned}
$$

where $Y_{p}=-\ln \left(-\ln \left(p_{i}\right)\right)$ is called the reduced variate. Once we have defined appropriate values of $p$ for each run-out ratio, we can solve for the parameters $u$ and $b$ using linear regression.

To define non-exceedance probabilities corresponding to each run-out ratio, we first rearrange the $N$ run-out ratios in increasing order so that

$$
\left(\frac{\Delta x}{x_{\beta}}\right)_{1} \leq\left(\frac{\Delta x}{x_{\beta}}\right)_{i} \leq\left(\frac{\Delta x}{x_{\beta}}\right)_{N} .
$$

For the corresponding non-exceedance probabilities, we use the empirical form obtained by McClung and Mears (1991),

$$
p_{i}=\frac{(i-0.4)}{N}
$$

where $i=1,2, \ldots, N$. 


\section{STATISTICAL RESULTS}

Second degree polynomials worked reasonably well as models of the 24 avalanche paths we surveyed. All but two yielded fits with standard deviations less than $6 \mathrm{~m}$ where the median was approximately $3.7 \mathrm{~m}$. Based on our data, we defined our reference point for $\theta_{\beta}=18^{\circ}$. Though we did not develop a firm physical basis for this definition, when higher values are used for $\theta_{\beta}$ the model is much more sensitive to measurement errors. Our data and definition of $\theta_{\beta}$ yield the statistics shown in Table 1 . As shown in Figure $1, \theta_{\mathrm{s}}$ and $\theta_{\mathrm{t}}$ represent the slopes of the starting and run-out zones, respectively.

Using regression on the linearized form of our extreme-value distribution, we obtain the parameters and corresponding $95 \%$ confidence limits on the mean shown in Figure 2. Since we have less than 30 data points, we used the $t$ distribution to calculate our confidence limits as discussed by Walpole and Myers (1972, section 8.3).

Though more than 24 avalanche paths were surveyed, some had to be eliminated because too few points were recorded to allow an accurate polynomial fit. One path, which had a large bench midway and was therefore inconsistent with the other paths, yielded a run-out ratio which far exceeded the run-out ratios of the other paths and was also eliminated.

\section{SOURCES OF UNCERTAINTY}

Since we expect a large range of weather patterns to occur over a $100 \mathrm{yr}$ period, we expect patterns which produce extreme avalanches to occur somewhere in that time frame. By measuring maximum avalanche runout distances, based on vegetation damage in the last $100 \mathrm{yr}$, we reduce the large variations in avalanche runout distances due to year-to-year weather patterns. At the same time, without completing a detailed tree-ring analysis at each path, we cannot be certain of the true time period that the vegetation damage has recorded, since the recovery rate for vegetation (trees) varies significantly between north and south aspects and also between higher and lower altitudes.

Of the avalanches recorded by vegetation damage, if we assume we have the maximum run-out distances for

Table 1. Descriptive statistics

Variable Mean Range

$\begin{array}{cccc}H(\mathrm{~m}) & 248 & 123 & 68-553 \\ \theta_{\mathrm{s}}(\mathrm{deg}) & 38 & 4.0 & 31-46 \\ \theta_{\mathrm{r}}(\mathrm{deg}) & 14.5 & 6.1 & 1.5-25 \\ \Delta x(\mathrm{~m}) & 66 & 102 & -87-340 \\ x_{\beta}(\mathrm{m}) & 432 & 221 & 140-982\end{array}$

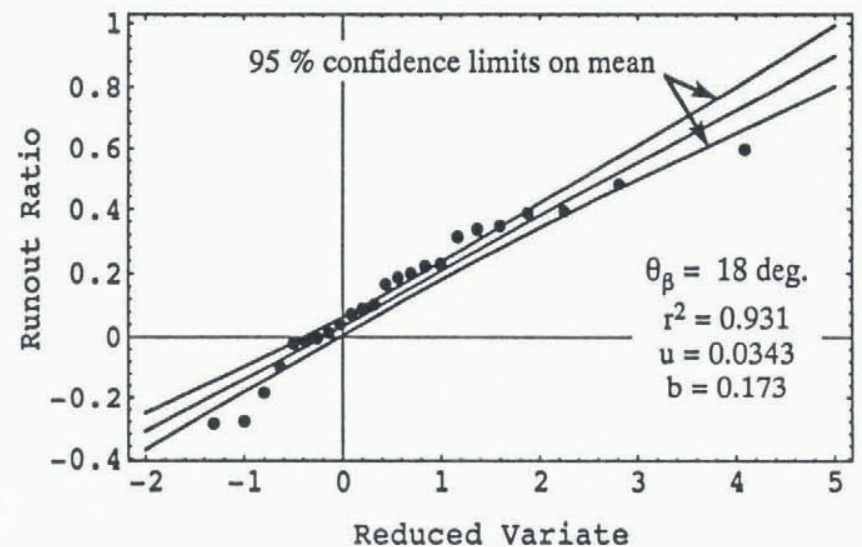

Fig. 2. Regression line for extreme-value distribution.

the last $100 \mathrm{yr}$, then most probably we are actually working with avalanche run-out distances that have return periods greater than $100 \mathrm{yr}$. For example, if we consider avalanches that have a return period of $1000 \mathrm{yr}$, then at each avalanche path there is a $9.5 \%$ chance we are working with a run-out distance that has a return period of $1000 \mathrm{yr}$. Using the binomial distribution, we estimate that approximately 2 of the 24 surveyed have a return period of $1000 \mathrm{yr}$, as long as they have not been over run by more extreme avalanches. Similarly, we would expect approximately 7 of the 24 surveyed to have a return period of $300 \mathrm{yr}$. Though these occurrences may appear to be a problem at first, they are not, since we would also expect similar occurrences in the next $100 \mathrm{yr}$. Also, since these occurrences are included in our data, they are also factored into our model.

Among the paths we survey, we have variations in flow resistance, path geometry, aspect, vertical drop, avalanche volume, etc. When we prepare to use the statistical model we must verify that the data set used to build the statistical model contained a sufficient number of avalanche paths with similar characteristics. Otherwise the model may be biased and yield poor predictions. Obviously, to develop a more accurate model it is necessary to work from a database of similar avalanche paths. On the other hand, to build a very general though conservative model, the data set must include a sufficient number of avalanche paths which bear all of the significant characteristics.

Unfortunately our data contain not only natural sources of variation but also errors and blunders introduced in the surveying or measuring process. The objective is to minimize the uncertainty in the model due to measurement errors and uncertainties. To do this we must obtain a feel for the effect each type of error might have on our model. To determine the model's sensitivity to these uncertainties we can use a Monte Carlo simulation as discussed in Press and others (1986). In essence, we superimpose normally distributed noise (errors) on our data and then record the resulting distribution of model parameters. From this distribution we can determine confidence limits relative to our measurement uncertainties.

As mentioned earlier, to survey the avalanche paths we first estimate the extreme limit of the starting zone or run-out zone, whichever is more convenient. We then 
record points along the avalanche path in terms of polar coordinates. If the slope appears to be relatively constant we take steps with distances (radii) as large as $50 \mathrm{~m}$, and where there are large variations in slope we shorten the recording distance accordingly. We measure the distance with a $50 \mathrm{~m}$ tape and the slope with an inclinometer (Brunton compass). This process is continued until we reach our estimate of the opposite extreme end of the avalanche path. Most of the paths had rocky cliff bands at the top which provided fairly definite starting points, but others began in large meadows or on convex break-over points which left the starting points open to judgement. The extreme run-out positions were usually better defined, but there were still cases where there was a choice between following a small off-shoot or stopping at the end of what appeared to be the bulk flow. Thus, based on our measuring technique, we have the following sources of uncertainty in our measurements:

(1) estimation of starting zone position;

(2) measurement of distance;

(3) measurement of slope; and

(4) estimation of extreme run-out position.

Rather than present an incomplete sensitivity analysis addressing these four sources of uncertainty, we will publish the complete sensitivity analysis in the proceedings of the 1992 International Snow Science Workshop held at Breckenridge, Colorado.

In addition to the previously mentioned sources of uncertainty, we also need to establish a quantitative measure of the quality of our model. In essence we should like to know if there is a good chance that we have chosen the right statistical model. To do this we have chosen to use the W test as discussed in Wadsworth (1990, section 6.7). Based on the size of our data set, with a $5 \%$ level of significance we should have a value for $\mathrm{W}$ between 0.369 and 0.979 . Our data yields a $\mathrm{W}$ value of 0.939 which implies our model satisfies the requirements at a $5 \%$ level of significance. However, our model does not satisfy the requirements at a $1 \%$ level of significance.

As a quick note of interest, McClung and Mears (1991, fig. 6) demonstrated the existence of length-scale effects in their data set from the Colorado Rockies. However, as shown in Figure 3, we did not see any similar behavior in our data. Also, if we consider the trend displayed in the paper by McClung and others (1989, fig. 2 ), we see that the expected values for run-out ratios increase as the mean size of the avalanches decreases. However, based on the fact that only five of our avalanches reached a local slope of $10^{\circ}$, our data indicates a reversal of this trend. The dominant factors may be hidden in the other natural sources of uncertainty.

\section{CONCLUSIONS}

Based on results obtained thus far, the extreme-value distribution model bears promise as a user-friendly tool for modeling even avalanches with small vertical drops; however, to be able to use this model more intelligently, natural sources of variation need to be further quantified.

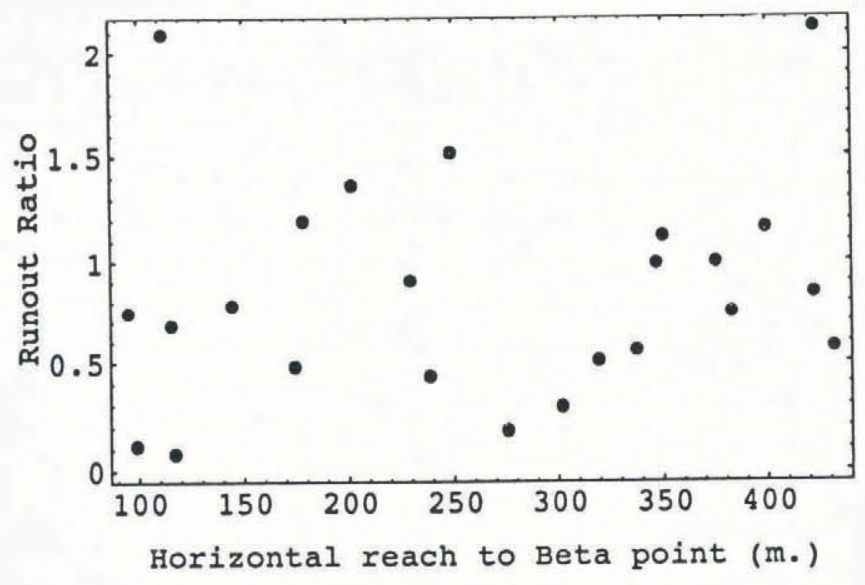

Fig. 3. No obvious length-scale effects.

With the small data set we have, no length-scale effects are apparent; therefore, length does not appear to be a significant source of variation for small avalanche paths.

The model developed here is based on a small data set and therefore cannot be considered reliable for estimating extreme avalanche run-out distances in the general case; however, the resulting extreme-value distribution does help shed light on a few characteristics of the model. In particular, since most of our extreme run-out points never reached a local slope angle of $10^{\circ}$, our results indicate that the models constructed by McClung and others are quite conservative when applied to avalanche paths in southwest Montana. To answer the questions that have been raised, more data must be collected on smaller avalanche paths $(H \leq 350 \mathrm{~m})$, then uncertainties should be further quantified statistically.

\section{ACKNOWLEDGEMENT}

The work reported here was funded by the Army Research Office under grant number DAAL 03-92-G0310. The authors wish to express their appreciation for ARO's support.

\section{REFERENCES}

Dent, J.D. and T.E. Lang. 1983. A biviscous modified Bingham model of snow avalanche motion. Ann. Glaciol., 4, 42-46.

Hutter, K., S.B. Savage and Y. Nohguchi. 1989. Numerical, analytical and laboratory experimental studies of granular avalanche studies. Ann. Glaciol., 13, 109-116.

Lang, R. 1991. An experimental and analytical study of gravity driven free surface flows of cohesionless granular media. (Ph.D. thesis, Technische Hochschule, Darmstadt.)

Lang, T.E. and R. L. Brown. 1980. Numerical simulation of snow avalanche impact on structures. U.S. Department of Agriculture. Forest Service. Research Paper RM-216.

Lang, T. E. and J. Dent. 1982. Review of surface friction, surface resistance, and flow of snow. Rev. Geophys. Space Phys., 20, 21-37. 
Lang, T. E., K. L. Dawson and M. Martinelli, Jr. 1979. Numerical simulation of snow avalanche flow. U.S. Department of Agriculture. Forest Service. Research Paper RM-205.

McClung, D. M. and K. Lied. 1987. Statistical and geometrical definition of snow avalanche runout. Cold Reg. Sci. Technol., 13(2), 107-119.

McClung, D. M. and A. I. Mears. 1991. Extreme value prediction of snow avalanche runout. Cold Reg. Sci. Technol., 19(2), 163-175.

McClung, D. M., A. I. Mears and P. Schaerer. 1989. Extreme avalanche run-out: data from four mountain ranges. Ann. Glaciol., 13, 180-184.
Martinelli, M., Jr, T.E. Lang and A. I. Mears. 1980. Calculations of avalanche friction coefficients from field data. 7. Glaciol., 26(94), 109-119.

Press, W., B. Flannery, S. Teukolsky and W. Vetterling. 1986. Numerical recipes. Cambridge, Cambridge University Press.

Wadsworth, H.J. 1990. Handbook of statistical methods for engineers and scientists. New York, McGraw-Hill.

Walpole, R. and R. Myers. 1972. Probability and statistics for engineers and scientists. New York, Macmillan.

The accuracy of references in the text and in this list is the responsibility of the authors, to whom queries should be addressed. 